\title{
A Deep Learning Framework for Human Activity Recognition Using Smartphone Data
}

\author{
J. Palimote' ${ }^{1}$ O.M.D. Georgewill ${ }^{2}$, L. Atu ${ }^{3}$ \\ Department of Computer Science, Kenule Beeson Saro-wiwa Polytechnic, Bori, River State, Nigeria ${ }^{1,2,3}$
}

\begin{abstract}
Smart phones are the most helpful apparatuses of our day-by-day life and with the propelling innovation; they get fit systematically to address user's issues and desires. To make these contraptions more useful and amazing, originators add new modules and gadgets to the equipment. Sensors has a major part in making cell phones more practical and mindful of the climate subsequently most smart phones accompany distinctive inserted sensors and this makes it conceivable to gather tremendous measures of data about the client's day by day life and exercises. The goal of Human Activity Recognition is to identify the activities performed by an individual from a given set of the information about him/her and his general environment. A great deal of exploration is being done in the field of Human Activity Recognition which human conduct is deciphered by reasoning highlights got from development, place, physiological signs and data from environments. The propose system presents a deep learning framework for human activity recognition using a smartphone data. The dataset was downloaded from kaggle.com. The dataset contains accelerometer and gyroscope data gotten from a Samsung Galaxy S2 smartphone. The accelerometer and gyroscope data is made up of different activities performed by an individual. The propose system uses keras framework and Theano as backend in build our model. After successful training, the proposed method had an accuracy of $99.06 \%$ on the $120^{\text {th }}$ epoch.
\end{abstract}

Keyword: Deep Learning Algorithm, Theano, Keras, Smartphone, Activities Recognition

\section{INTRODUCTION}

Smart phones are the most helpful apparatuses of our day-by-day life and with the propelling innovation; they get fit systematically to address user's issues and desires. To make these contraptions more useful and amazing, originators add new modules and gadgets to the equipment. Sensors has a major part in making cell phones more practical and mindful of the climate subsequently most smart phones accompany distinctive inserted sensors and this makes it conceivable to gather tremendous measures of data about the client's day by day life and exercises. Accelerometer and gyrator sensors are among these gadgets as well. Accelerometer has been a standard equipment for practical to all smart phone producers. As its name proposes accelerometer quantifies the adjustment in speed; not simply the speed [1].

Information recovered from accelerometer might be handled to distinguish unexpected changes in development. Another sensor that has been a standard equipment for cell phones is whirligig, which estimates direction by utilizing gravity. Signals recovered by gyrator can be handled to distinguish position and arrangement of the gadget. Since there is an important distinction of attributes between pieces of information recovered from these sensors, numerous highlights could be created from these sensors information to decide movement of the individual that is conveying the gadget. Order of smart phone user exercises has been engaged in various investigations. Concentrated on human activity recognition with quickening agent signals. [2] Attempted to characterize movement relying upon wearable different gyrator and accelerometers

The goal of Human Activity Recognition is to identify the activities performed by an individual from a given set of the information about him/her and his general environment. A great deal of exploration is being done in the field of Human Activity Recognition which human conduct is deciphered by reasoning highlights got from development, place, physiological signs and data from environments. Ecological and sensors, which are worn by the individual, creates the data, which is utilized to decipher the movement. Great exactness can be gotten from sensors, which are worn in midsection, wrist, chest and thighs. In any case, these sensors are very awkward and cannot give long haul arrangements. Cell phones have raised gigantic exploration openings in human-focused applications [3]

The most recent cell phones accompanied different implanted sensors like mouthpieces, cameras, accelerometers, spinners, and so forth, these regularly accessible gadgets give programmed and unhindered observing of everyday life 
exercises separated from communication administrations. Human Activity Recognition is essential for a lot bigger idea known as setting mindful figuring or omnipresent. Universal processing manages its responsibility similarly as Human Activity Recognition, which is by gathering information from clients and helping them [4].

\section{RELATED WORKS}

Recognition of Daily Human Activity Using an Artificial Neural Network and Smart watch [5] proposed two models, in which one uses just increasing speed sensor information and other uses area data notwithstanding quickening sensor information. Before include extraction, the increasing speed sensor information is partitioned into time portions, which is supposed to be fleeting division. To deal with spilling of information, sliding window method is utilized. The segment vector of the time window is used as the commitment to the classifier. Using territory information, a logically express and organized region based classifier can be associated. The area data isn't assembled from the structure and it is gotten from various types of exercises. In the Artificial Neural Network (ANN) classifier, the Xavier and ReLU are generally used to diminish learning time in the field of AI. The learning rate is set to 0.01 , and the Adam smoothing out specialist is used since it is known to achieve incredible results speedy. At last, the model with area data shows a precision of $95 \%$ and the model without area data does $90 \%$ exactness.

Smartphone Sensor-Based Activity Recognition by Using Machine Learning and Deep Learning Algorithms [6] proposed a method for activity recognition in two methods. In method 1 , accelerometer gives crude sensor information, which perceives the activities and the course of cell phones showing the gyrator faculties rotational developments, which are huge to recognize by individuals. In method 2, highlight extraction strategy is performed for the detected information. AI models worked for activity recognition, by then the significant learning model reliant on convolutional neural framework. The outcomes are exhibited to demonstrate consistency; it expected that the extent of exercises acknowledgment could be stretched out with the usage of accumulated data with multi-class dataset. The trial results show that SVM performs preferred in all plan over FLD as far as exactness. From the outcomes, it will in general be contemplated that accelerometer sensor (A-sensor) perusing offer more contrasted with G-sensor perusing (Gyroscope sensor) however can build the precision of the recognition by using both AI and significant learning counts.

Human Activity Recognition Using Smartphones [7] proposes several supervised machine learning techniques, which are, Decision trees, Support Vector Machines, K-nearest neighbors (KNN) and ensemble technique such as, Boosting, Bagging and Stacking. For grouping, twofold choice tree is utilized in which 53.1\% of exactness. At the point when as far as possible is expanded to 100, the precision is expanded to $94.4 \%$. Support Vector machines gives a precision of $99.4 \%$ that utilizes hyper dimensional planes. K-NN furnishes an expanded productivity of $97.1 \%$ with $\mathrm{k}$ worth is set to 3. In Ensemble methods there are distinctive promotion in which boosting strategy has various structures. AdaBoost utilizes misjudged likelihood and orders with a precision of $97.4 \%$. The alternate way is stowing which is utilized to get goal results from machine learning approaches with proficiency of $98.1 \%$. Third way is stacking in which 30 stack classifiers are applied and anticipated an exactness of $98.6 \%$.

A Study on Human Activity Recognition Using Accelerometer Data from Smartphone [8] carried out analysis on various exercises of an individual, utilizing which an arrangement model is assembled dependent on the feature extraction. In Weka toolbox, Multilayer Perceptron, Random woodland, LMT, SVM, Simple Logistic and LogitBoost are looked at as an individual and joined classifier then it was approved utilizing K-overlay cross approval. The acknowledgment is dissected as versatile close by and pocket position. The proficiency is gotten as SVM gives better exactness close by and Random woods directs most elevated precision. Cell phone close by position, the combination of Multilayer Perceptron, Logit Boost and SVM classifier yields an exactness of 91.15\% however then in pocket position, Multilayer Perceptron, Random Forest and Simple Logistic with 90.34\% precision.

Activity Recognition in Egocentric video using SVM, KNN and Combined SVM and KNN Classifiers [9] proposed a recognition framework wherein another advanced low-pass channel was planned to segregate the part of gravity speeding up from that of body quickening in the crude information. They prepared and tested their framework in an experiment with numerous human subjects in true conditions. A few classifiers were tried utilizing different factual highlights. High-recurrence and low-recurrence segments of the information were considered. They chose five classifiers each offering great execution for perceiving their set off exercises and researched how to join them into an ideal arrangement of classifiers. They inferred that utilizing the normal of probabilities as the combination strategy could arrive at a general exactness pace of $91.15 \%$.

A robust human activity recognition system using smartphone sensors and deep learning [10] present a smart phone inertial sensors-based methodology for human activity recognition. Productive columns are first extricated from raw information. The features incorporate mean, median, autoregressive coefficients, and so forth. The features are 
additionally prepared by a part head segment investigation (KPCA) and direct discriminant examination (LDA) to make them more powerful. At last, the features are prepared with a Deep Belief Network (DBN) for effective activity recognition. The proposed approach was contrasted and customary action recognition approaches, for example, regular multiclass Support Vector Machine (SVM) and Artificial Neural Network (ANN) where it outflanked them.

Real-time human activity recognition from accelerometer data using Convolutional Neural Networks [11] presented a client autonomous deep learning-based methodology for online human movement recognition. They propose utilizing Convolutional Neural Networks for nearby component extraction along with straightforward factual highlights that save data about the worldwide type of time arrangement. Moreover, they explore the effect of time arrangement length on the acknowledgment precision and breaking point it up to $1 \mathrm{~s}$ that makes conceivable persistent constant action grouping. The precision of the proposed approach is assessed on two generally utilized WISDM and UCI datasets that contain marked accelerometer information from 36 and 30 clients separately, and in cross-dataset test. The outcomes show that the proposed model exhibits cutting edge execution while requiring low computational expense and no manual component designing.

\section{DESIGN METHOLOGY}

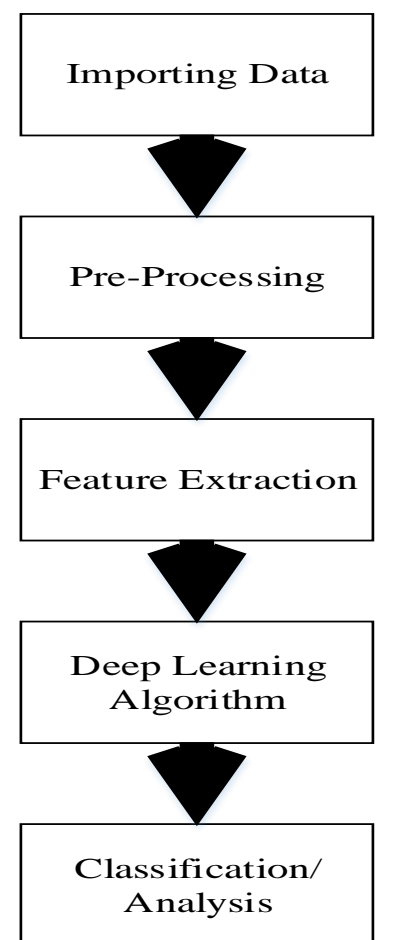

Figure 1: Architecture of the propose system design

Importing Data: The dataset was downloaded from kaggle.com. The dataset contains accelerometer and gyroscope data gotten from a Samsung Galaxy S2 smartphone. The accelerometer and gyroscope data is made up of different activities performed by an individual.

Pre-processing: Pre-processing has to do with the conversion of some alphabetical values to 0 s and 1 s for easy fitting and better performance of the Deep learning algorithm. The dataset was preprocessed by scaling some values using standard scaler and also by converting the activities to numerical values using an encoder.

Feature Extraction: This has to do with remover of some unwanted features, and also by bringing all features to a pre-defined function.

Deep Learning Algorithm: The deep learning algorithm adopted in this research is a feed forward neural network with four dense layer, and a total of 563 input neurons. For the input activation, we use tanh and for the output activation, we use softmax. 


\section{DOI 10.17148/IJARCCE.2021.10201}

Classification/Analysis: After successful building and training of our model, we will check the performance of our model by using a confusion matrix for evaluation and analysis.

Table 1: Confusion Matrix Evaluation Formula

\begin{tabular}{|l|c|}
\hline Definition & Formula \\
\hline Accuracy & $T P+T N$ \\
\cline { 2 - 2 } & $\frac{T P+F N+T N+F P}{1 \text {-accuracy }}$ \\
\hline Error & $\frac{T P}{T P+F N}$ \\
\hline Recall & $\frac{\mathrm{TP}}{\mathrm{TP}+\mathrm{FP}}$ \\
\hline Frecision & $\frac{2 \text { Precison } \times \text { Recall }}{\text { Precision }+ \text { Recall }}$ \\
\hline
\end{tabular}

\section{DISCUSSION OF RESULTS}

The proposed system uses a smartphone dataset, which contains some accelerometer and gyroscope information of the activities performed by an individual using a Samsung galaxy S2 smartphone. The dataset was preprocessed by converting the character values in the activities columns to numerical values (0-5) using a label Encoder function for easing fitting and training of our model. The character values converted are "Walking", "Walking_Upstairs", "Walking_Downstairs", "Sitting", "Standing", "Laying". Feature extraction was used in dropping some features, and using just the features that are need for the training of our model .Therefore, bringing all features to a predefined dimension. After these processes, we trained our model by using the following threshold functions and parameters. input_dimension $=563$, activation $=$ 'tanh', init $=$ 'lecun_uniform', w_regularizer $=12 * 0.001$, Dropout $=0.5,0.2$, learning_rate $=.13$, momentum $=.9$, decay $=4 \mathrm{e}-3$, optimizer $=$ 'sgd', loss $=$ 'binary_crossentropy', batch_size $=50$, epochs $=120$. The proposed method uses Keras and Theano for backend. After successful training, the proposed method had an accuracy of $99.06 \%$ on the $120^{\text {th }}$ epoch, which can be seen in figure 4 and 5 .

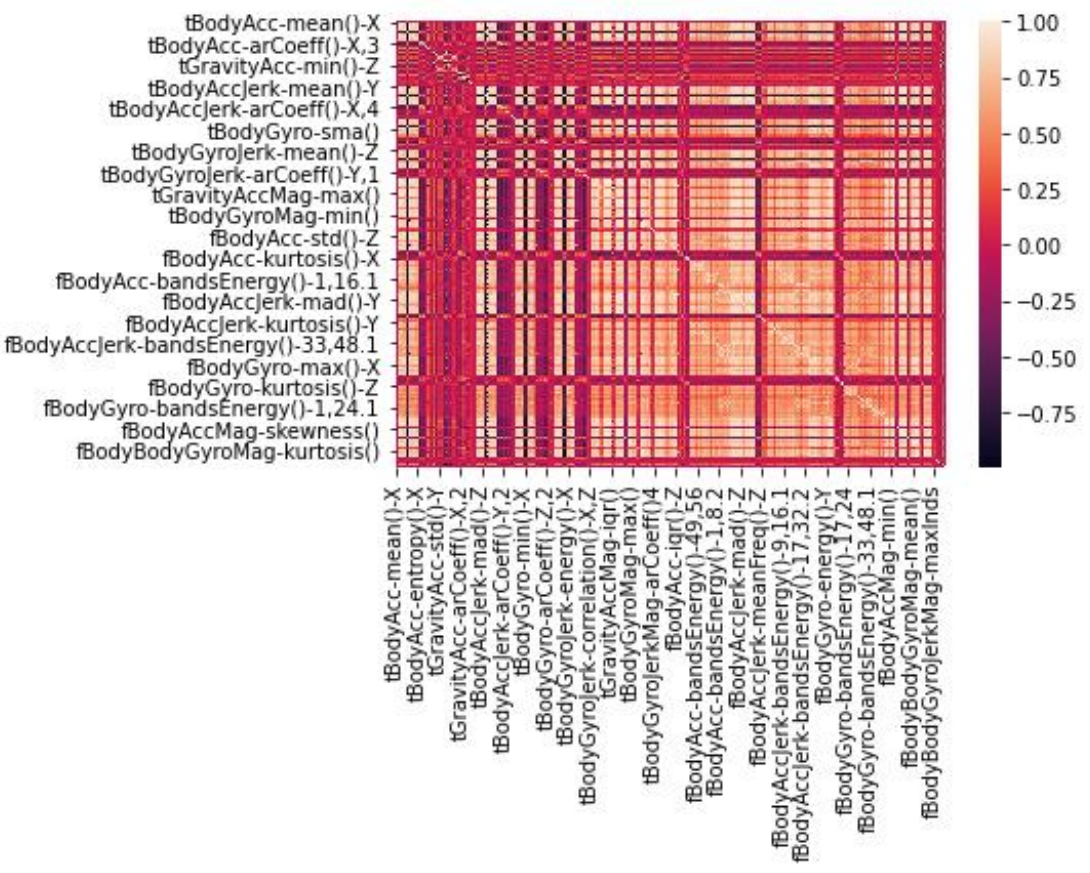

Figure 2 The correlation matrix of the dataset 


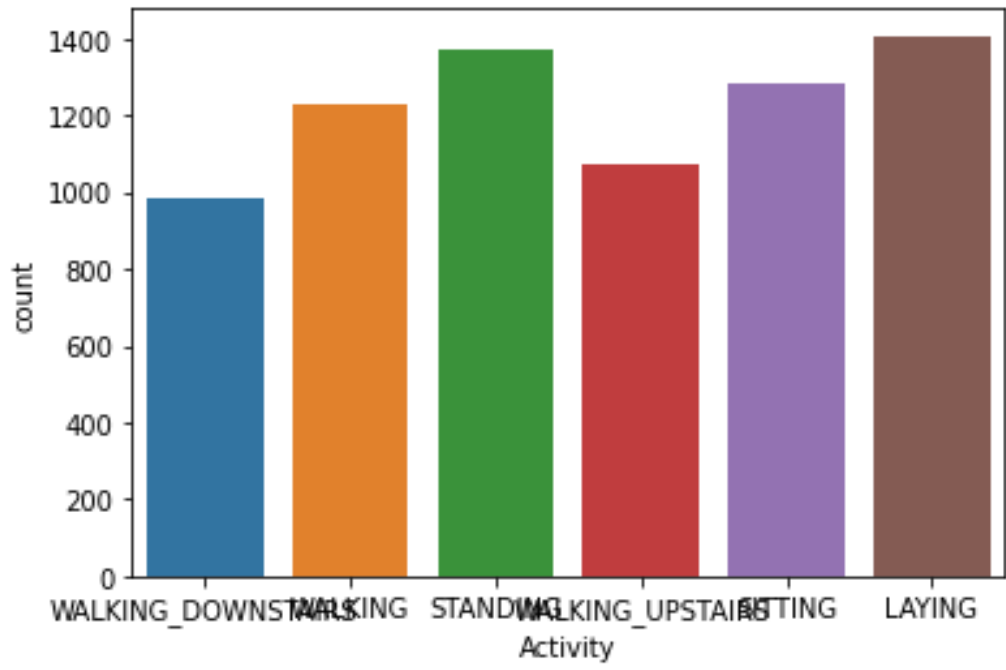

Figure 3: Histogram of the number of activities that are carried out in the dataset. These activities are walking, walking downstairs, standing, walking upstairs, sitting and laying

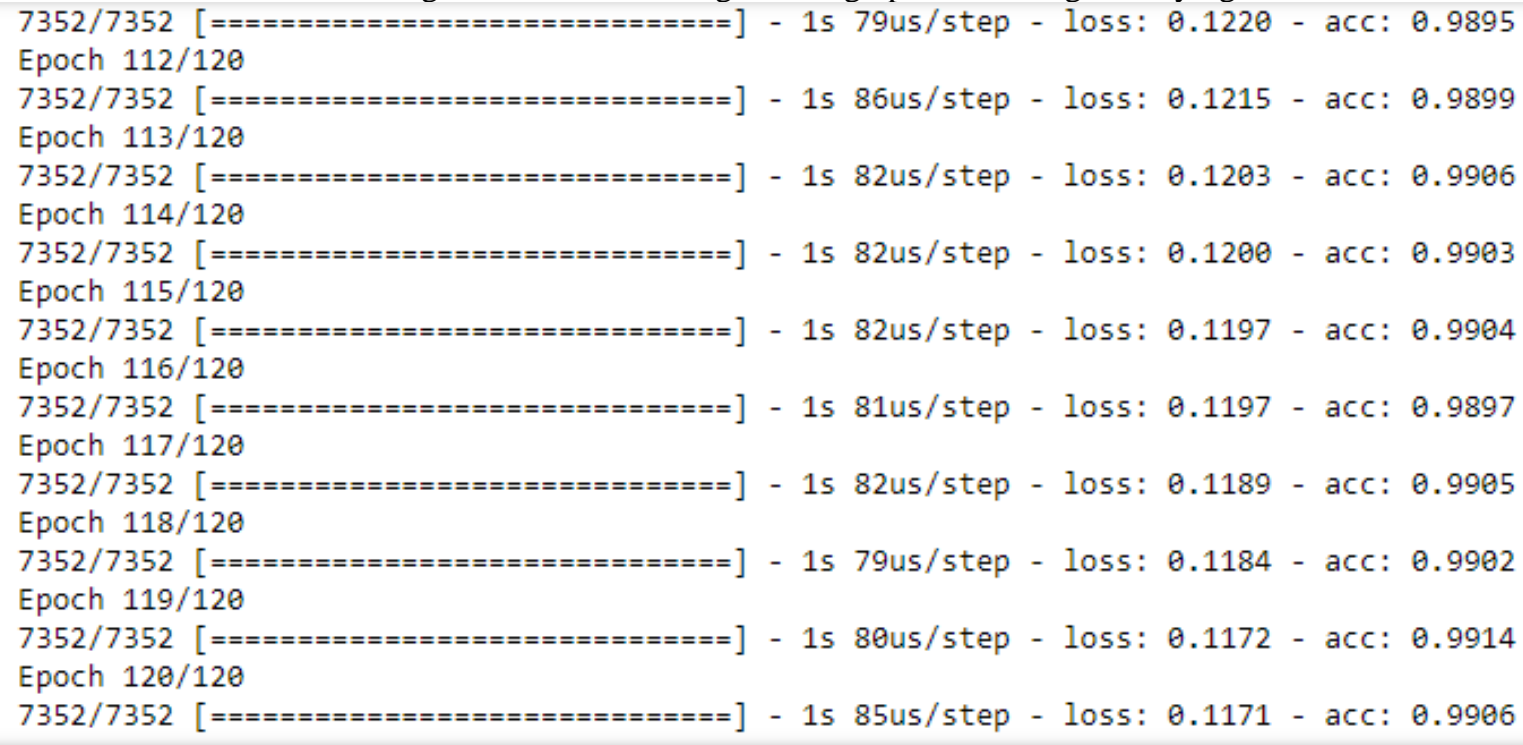

Figure 4: The training process starting from the 112 to 120 epoch values. The figure shows the time, which is defined by seconds in completing one step, and also the loss and accuracy value at each of the step. 
Vol. 10, Issue 2, February 2021

DOI 10.17148/IJARCCE.2021.10201

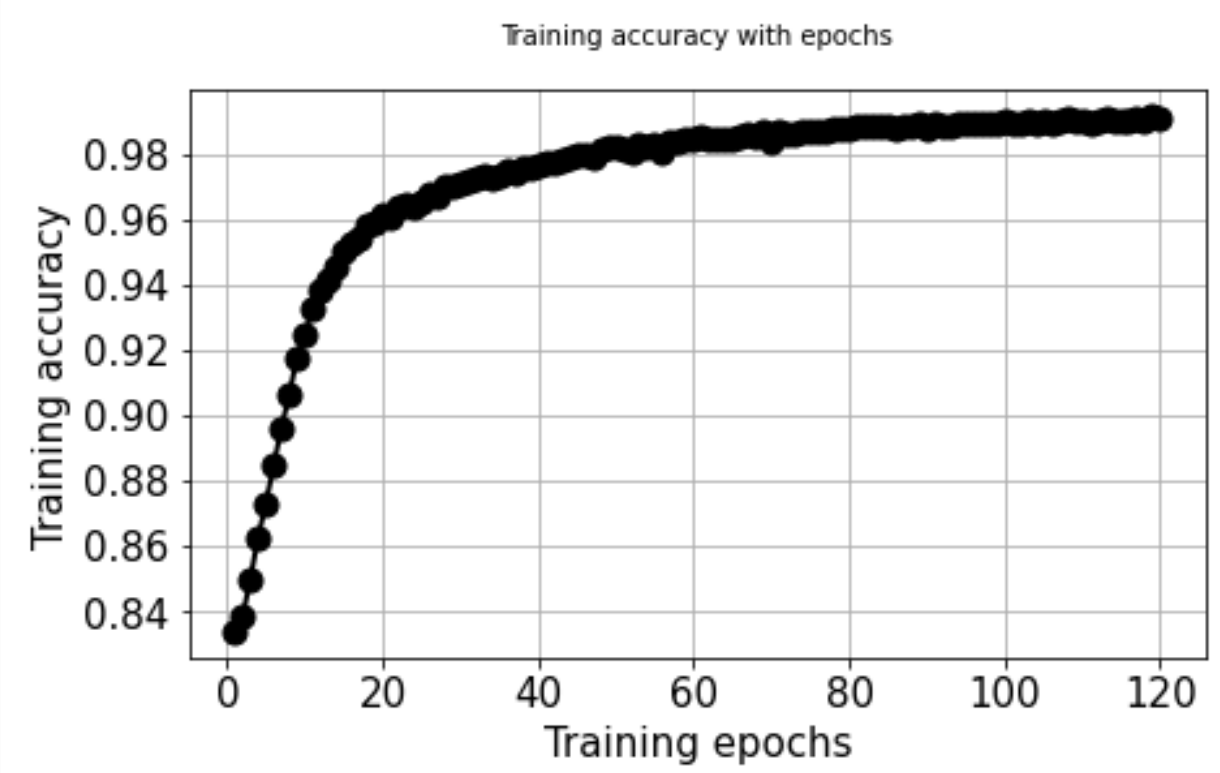

Figure 5 Plot between training accuracy and training epoch Training loss with epochs

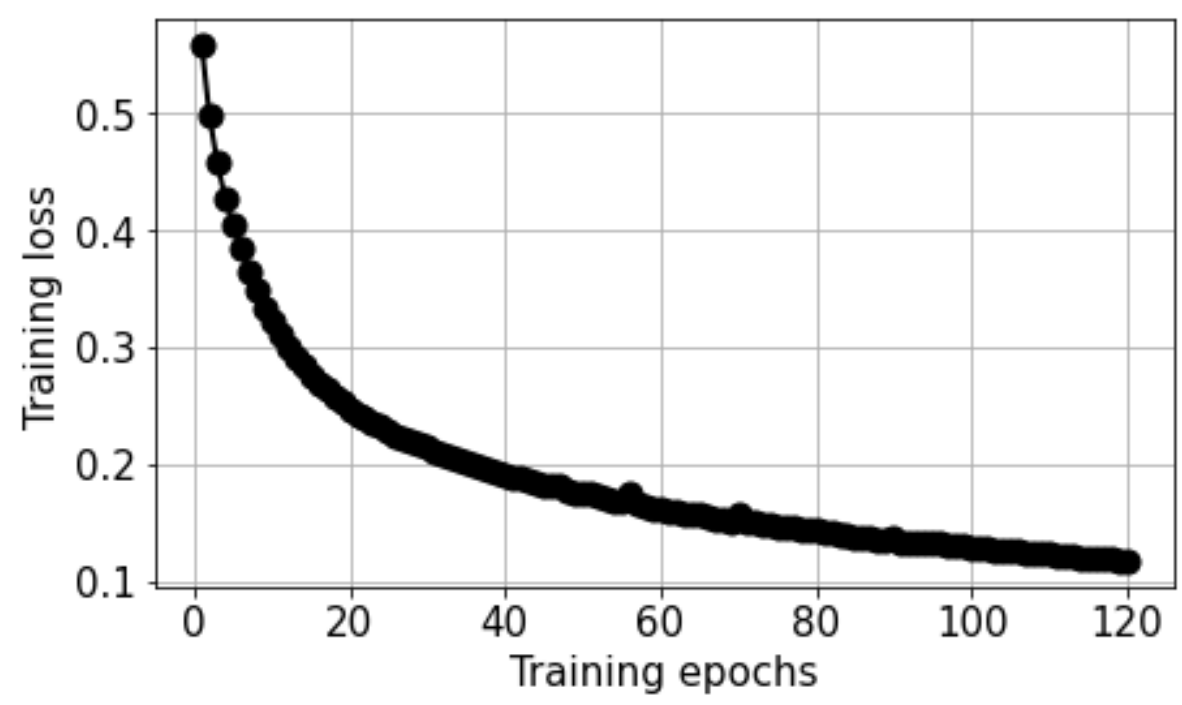

Figure 6 Plot between training loss and training epoch

\section{CONCLUSION}

Smart phones are the most helpful apparatuses of our day-by-day life and with the propelling innovation; they get fit systematically to address user's issues and desires. To make these contraptions more useful and amazing, originators add new modules and gadgets to the equipment. Sensors has a major part in making cell phones more practical and mindful of the climate subsequently most smart phones accompany distinctive inserted sensors and this makes it conceivable to gather tremendous measures of data about the client's day by day life and exercises. The proposed system design uses a smartphone dataset, which contains some accelerometer and gyroscope information of the activities performed by an individual using a Samsung galaxy S2 smartphone. The dataset was preprocessed by converting the character values in the activities columns to numerical values (0-5) using a label Encoder function for 


\section{International Journal of Advanced Research in Computer and Communication Engineering}

Vol. 10, Issue 2, February 2021

\section{DOI 10.17148/IJARCCE.2021.10201}

easing fitting and training of our model. The character values converted are "Walking", "Walking_Upstairs", "Walking_Downstairs", "Sitting", "Standing", "Laying". Feature extraction was used in dropping some features, and using just the features that are need for the training of our model .Therefore, bringing all features to a predefined dimension. This system uses Theano as backend and keras in building our model. After successful training, the proposed method had an accuracy of $99.06 \%$ on the $120^{\text {th }}$ epoch, which can be seen in figure 4 and 5 .

\section{REFERENCES}

[1]. Bayat A., Pomplun M., and Tran D.A., A Study on Human Activity Recognition Using Accelerometer Data from Smartphones, Elsevier Procedia Computer Science - MobiSPC, pp.1-8, 2014.

[2].F. Attal, S. Mohammed, M. Dedabrishvili , F. Chamroukhi, L. Oukhellou, Y. Amirat, "Physical Human Activity Recognition Using Wearable Sensors "Academic Editor: Vittorio M.N. Passaro, 2015.

[3]. Davide Anguita, Alessandro Ghio, Luca Oneto, Xavier Parra and Jorge L. ReyesOrtiz, "A Public Domain Dataset for Human Activity Recognition Using Smartphones", European Symposium on Artificial Neural Networks, 24-26 April 2013.

[4]. Adil Mehmood Khan, Young-Koo Lee, Sungyoung Y. Lee, and Tae-Seong Kim,A triaxial accelerometer-based physical -activity recognition via augmented-signal features and a hierarchical recognizer, Information Technology in Biomedicine, IEEE transactions on information technology in biomedicine, Volume 14, NO. 5, September 2010.

[5]. Min-Cheol_Kwon and Sunwoong_Choi, "Recognition of Daily Human Activity Using an Artificial Neural Network and Smart watch",Wireless Communications and Mobile Computing, 2018.

[6].Qingzhong Liu, Zhaoxian Zhou, Sarbagya Ratna Shakya, Prathyusha Uduthalapally, Mengyu Qiao, and Andrew H. Sung, "Smartphone SensorBased Activity Recognition by Using Machine Learning and Deep Learning Algorithms" International Journal of Machine Learning and Computing, Vol. 8, No. 2, April 2018.

[7]. Erhan BÜLBÜL, Aydın Çetin and İbrahim Alper DOĞRU, "Human Activity Recognition Using Smartphones", IEEE, 978-1-5386-4184, 2018.

[8].Akram Bayat*, Marc Pomplun, Duc A. Tran, "A Study on Human Activity Recognition Using Accelerometer Data from Smartphones", Department of Computer Science, University of Massachusetts, Boston, 100 Morrissey Blvd Boston, MA 02125, USA, Elsevier Procedia Computer Science, $, \mathrm{Vol} 34,450-457,2014$.

[9]. K.P. Sanal Kumar and R. Bhavani, "Activity Recognition in Egocentric video using SVM, KNN and Combined SVM KNN Classifiers", IOP Conference Series: Materials Science and Engineering, 2017 Sandeep Kumar Polu, "Human Activity Recognition on SmartPhones.

[10]. M.M. Hassana, Z. Uddin A. Mohamed A. Almogrena "A robust human activity recognition system using smartphone sensors and deep learning", Future Generation Computer Systems, Vol.81, pp. 307-313 2018.

[11]. Andrey Ignatov, "Real-time human activity recognition from accelerometer data using Convolutional Neural Networks", Applied Soft Computing, Vol. 62, pp.915-922, 2018 